\title{
A space weather index for the radiation field at aviation altitudes
}

\author{
Matthias M. Meier* and Daniel Matthiä \\ German Aerospace Center, Institute of Aerospace Medicine, Linder Höhe, 51147 Köln, Germany \\ *Corresponding author: Matthias.Meier@dlr.de
}

Received 24 January 2014 / Accepted 28 March 2014

\begin{abstract}
The additional dose contribution to the radiation exposure at aviation altitudes during Solar Particle Events (SPEs) has been a matter of concern for many years. After the Halloween storms in 2003 several airlines began to implement mitigation measures such as rerouting and lowering flight altitudes in response to alerts on the NOAA S-scale regarding solar radiation storms. These alerts are based on the integral proton flux above $10 \mathrm{MeV}$ measured aboard the corresponding GOES-satellite which is operated outside the Earth's atmosphere in a geosynchronous orbit. This integral proton flux has, however, been proved to be an insufficient parameter to apply to the radiation field at aviation altitudes without an accompanying analysis of the shape of the energy spectrum. Consequently, false alarms and corresponding disproportionate reactions ensued. Since mitigating measures can be quite cost-intensive, there has been a demand for appropriate space weather information among responsible airline managers for about a decade. Against this background, we propose the introduction of a new Space Weather index D, based on dose rates at aviation altitudes produced by solar protons during solar radiation storms, as the relevant parameter for the assessment of corresponding radiation exposure. The Space Weather index D is a natural number given by a graduated table of ranges of dose rates in ascending order which is derived by an equation depending on the dose rate of solar protons.
\end{abstract}

Key words. solar particle events - aviation - radiation exposure of aircrew - space weather index

\section{Introduction}

The radiation field at aviation altitudes is generated by interactions of primary cosmic radiation of solar and galactic origin with the constituents of the Earth's atmosphere. Galactic cosmic radiation provides the main component due to the continuous impingement of high-energy charged particles which have to overcome three protective mechanisms in order to contribute to this radiation field, namely: the interplanetary magnetic field (IMF), the Earth's magnetosphere and the upper atmosphere. The IMF is modulated by solar activity which varies over an approximately 11-year cycle. In addition to modulating the galactic component during the solar cycle, solar activity can affect the radiation field at aviation altitudes in different ways. Solar particles with energies high enough to penetrate sufficiently deeply into the atmosphere can, during rare events, temporarily increase the corresponding dose rates by several orders of magnitude. In contrast to these rare events, increased solar proton fluxes generally lead to decreased radiation intensities at aviation altitudes due to the additional magnetic shielding from the high-energetic galactic cosmic radiation component when the solar particles are not energetic enough to contribute to the radiation field themselves. This effect can be usually observed about one to two days after the initial Solar Particle Event (SPE) and is known as a Forbush-Decrease.

On 28th October 2003 during a period of turbulent solar activity also known as the Halloween Storms, the Federal Aviation Administration (FAA) issued a radiation alert, which led to a rerouting and lowering of flight altitudes by several American and European airlines in order to mitigate a potential increase in dose rates between 29th and 31st October 2003 (Lieber 2003; US Department of Commerce, Service Assessment 2004). This solar proton alert was based on an assessment of the additional radiation exposure having a trigger threshold of $20 \mu \mathrm{Sv} / \mathrm{h}$ at any altitude between $30,000 \mathrm{ft}$. $(9.1 \mathrm{~km})$ and $70,000 \mathrm{ft}$. $(21.3 \mathrm{~km})$ for each of three consecutive 5 -min periods without geomagnetic shielding, i.e. for polar routes (Copeland et al. 2005). The spectrum of the impinging solar protons was derived from the particle fluxes measured aboard the GOES-11 satellite. A more detailed analysis of the situation between the 28th and 31st October will be given in the following section.

Since the Halloween Storms, airlines and aircraft operators have been interested in timely and accurate information concerning significant increases in the radiation intensity at aviation altitudes in order to be able to mitigate potential hazardous radiation effects on humans and on avionics, e.g. by temporarily reducing flight altitudes. This mitigation measure, however, results in higher cost, fuel consumption and atmospheric pollution. Therefore, accurate information about the period and radiation level involved is required in order to reduce the radiation exposure to a level which is as low as is reasonably achievable.

\section{The S-scale for solar storms}

After the Halloween Storms, a service assessment team was formed to examine the space environment warning and forecast services provided by the NOAA Space Environment Center (now the Space Weather Prediction Center, US Department of Commerce, Service Assessment 2004). An important finding was that some airlines had established their action level based on at least an S3 solar storm warning. Reactions to such warnings consist of rerouting polar flights and lowering flight altitudes. 
Begin: 2003

GOES11 Proton Flux (5 minute data) Oct 280000 UTC

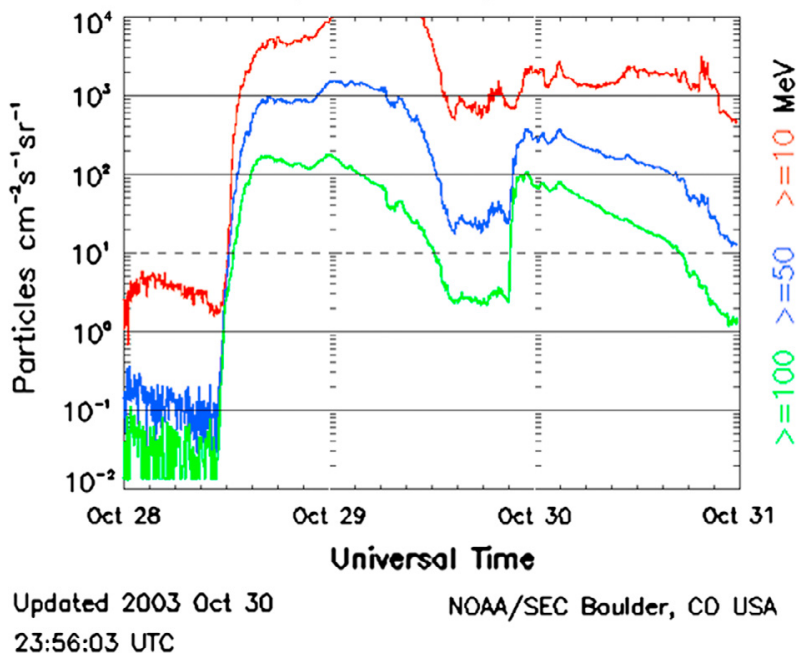

Fig. 1. GOES 5-min averaged integral proton flux (protons $\times \mathrm{cm}^{-2} \times \mathrm{s}^{-1} \times \mathrm{sr}^{-1}$ ) as measured by the SWPC primary GOES satellite for energy thresholds of $\geq 10$ (red line), $\geq 50$ (blue line), and $\geq 100 \mathrm{MeV}$ (green line) between 28th and 31st October 2003. SWPC's proton event threshold is 10 protons $\times \mathrm{cm}^{-2} \times$ $\mathrm{s}^{-1} \times \mathrm{sr}^{-1}$ at $\geq 10 \mathrm{MeV}$ (http://www.swpc.noaa.gov/rt plots/pro 3d.html).

The S-scale for solar storm warnings was established in 1999 as a part of the NOAA Space Weather Scales, akin to the hurricane scale or as a sort of Richter scale, in order to provide space weather information for the non-expert (Poppe 2000; Poppe \& Jorden 2006). The underlying observed physical quantity is the integral flux of protons above $10 \mathrm{MeV}$ measured aboard the currently operational GOES-satellites, which is given in proton flux units (pfu), defined by the number of protons $\times \mathrm{cm}^{-2} \times \mathrm{s}^{-1} \times \mathrm{sr}^{-1}$. Usually, the background level is of the order of $0.1 \mathrm{pfu}\left(10^{-1}\right.$ particles $\left.\times \mathrm{cm}^{-2} \times \mathrm{s}^{-1} \times \mathrm{sr}^{-1}\right)$, during quiet times, i.e. without increased solar proton emission. Alerts are issued when the measured proton fluxes exceed defined thresholds of $10^{\mathrm{n}} \mathrm{pfu}$, where $\mathrm{n}$ denotes the corresponding level on the S-scale, i.e. 10 pfu corresponds to the threshold of an S1 alert which is the lowest warning level. A value of $100 \mathrm{pfu}$ corresponds to the threshold of an S2 alert and so on. A graph showing the temporal development of the proton flux with energies above $10 \mathrm{MeV}$ measured aboard the currently operational GOES-satellites is available on the NOAA web page in near real time (http://www.swpc.noaa.gov/ rt plots/pro 3d.html). A corresponding graph for the situation between 28th and 31st October 2003 is presented in Figure 1. On 28th October at noon there was a sudden strong increase in the particle flux from a slightly elevated background level of approximately 2 pfu to more than 5000 pfu. This flux increased further over the next couple of hours and exceeded the threshold of an S4 alert (10,000 pfu) at around midnight. Thereafter, this warning situation lasted for about $12 \mathrm{~h}$. In this context, it is worth noting that the fraction of particles with energies above $100 \mathrm{MeV}$ given by the green line in Figure 1 is less than about $1 \%$ of the total flux of particles above $10 \mathrm{MeV}$ which is given by the red line and provided the trigger for the corresponding solar radiation storm alert.

Direct information about the variation of the radiation intensity inside the Earth's atmosphere can be obtained from neutron monitors on the ground. Consequently, the effect of the Earth's magnetic field on the radiation intensity in the atmosphere can be investigated using neutron monitors at different geomagnetic locations. The variation of the count rates of the neutron monitor in Oulu (Finland) during the solar radiation storm period between 28th and 31st October 2003 is shown in Figure 2. In contrast to the strong increase in the primary proton flux measured by GOES (Fig. 1), the neutron monitor count rates increased by about $5 \%$ only and had already fallen below the background level some $9 \mathrm{~h}$ later. An event that can be observed using a neutron monitor on the ground is generally referred to as a GLE (Ground Level Enhancement). GLEs have been enumerated since 1942; the event of 28th October 2003 is in particular referred to as GLE 65 (http://neutronm.bartol.udel.edu/ $\sim$ pyle/GLE_List.txt). The decrease in the count rates down to about $22 \%$ below the initial background level during the 29th October indicated the presence of additional shielding from galactic cosmic radiation due to the contribution to the interplanetary magnetic field (IMF) associated with the high fluxes of solar protons (Forbush Effect).

A further source of information is available through measurements from inflight radiation monitors. A detailed and comprehensive analysis of the effect of the solar radiation environment on dose rates at aviation altitudes based on measurements with a TEPC (Tissue Equivalent Proportional Counter) showed only a comparatively slight increase in dose rates of about $30 \%$ during the course of GLE 65 and a decrease of the same order of magnitude during the following ForbushDecrease (Beck et al. 2005). These findings are supported by measurements made using different dosimetric equipment during a flight from Los Angeles (LAX) to New York (JFK) on 29th October 2003 (Getley 2004). These measurements clearly demonstrate that the radiation field in the atmosphere, and at aviation altitudes in particular, not only did not follow the behaviour of the proton flux at the position of the GOES-11 spacecraft but even showed an opposing trend during the Forbush-Decrease.

The observation of a strong increase in proton fluxes at the position of the GOES-satellite in connection with comparatively small increases in dose rates at aviation altitudes and even a decrease due to the Forbush Effect can be explained by the distribution of the energies of the incoming solar protons, i.e. the corresponding energy spectrum. While the trigger for NOAA S-scale alerts is based on proton fluxes above $10 \mathrm{MeV}$, much higher energies of the impinging particles are required to produce a significant contribution to the radiation field at aviation altitudes due to atmospheric shielding of both the solar protons and the secondary particles they generate in the upper atmosphere. Calculations using the PANDOCA radiation transport model have shown that only protons above about $600 \mathrm{MeV}$ are able to reach the upper airspace and contribute significantly to the corresponding dose rate (Matthiä et al. 2014). Even though protons of lower energies can indirectly contribute to the radiation exposure through secondary particle production, especially of neutrons, the relevant primary particle energies are more than an order of magnitude greater than the $10 \mathrm{MeV}$ threshold used for the S-scale alert. As the energy spectrum of the solar protons is usually well described by a power law the number of particles decreases drastically with increasing energy. In consequence, the contribution of solar protons above the energy threshold, required to produce radiation exposure at aviation altitudes, is usually several orders of magnitude smaller than the total flux above $10 \mathrm{MeV}$. Thus, although a high total proton flux is a necessary condition for an increase in radiation exposure at aviation altitudes, it is not 
M.M. Meier and D. Matthiä: A space weather index for the radiation field at aviation altitudes

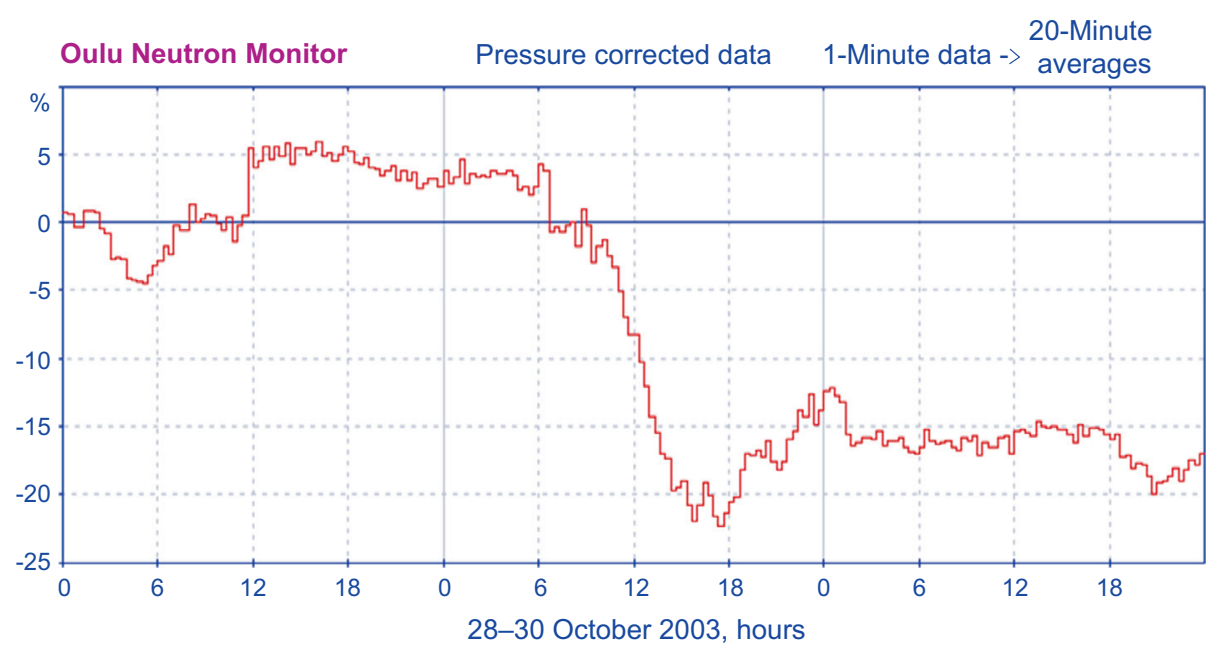

Fig. 2. Variation of the count rates of the Oulu neutron monitor between 28th and 31st October 2003 (http://cosmicrays.oulu.fi/).

a sufficient condition which requires in addition an analysis of the shape of the energy spectrum of the impinging particles. Higher energies in the range above $1 \mathrm{GeV}$ of primary cosmic radiation are measured by ground-based neutron monitors and there is no indication that a significant increase in the corresponding dose rates at aviation altitudes can occur without a strong GLE measured by these detectors. Consequently, a warning system for the radiation exposure at flight altitudes which only depends on the proton flux above $10 \mathrm{MeV}$ outside the Earth's atmosphere is prone to generate many false alarms.

The shortcomings of the S-scale for applications which require more specific information on highly energetic particles had already been discussed by the developers when the NOAA Space Weather Scales were introduced (Poppe 2000). In this context, it is important to clearly point out that the proton fluxes provided by NOAA's GOES satellites still constitute a very useful tool for forecasting ionospheric disturbances which might also have an impact on aviation through disrupting HF communications. Furthermore, the proton flux data provide valuable information for the assessment of the radiation exposure of satellites as well as for manned space missions. It should be kept in mind that the radiation measuring instruments aboard the GOES-spacecraft were devised for these purposes and the restricted applicability of the associated S-scale for an assessment of the radiation exposure at flight altitudes does not reduce the benefit of the corresponding proton flux data for achieving these goals.

\section{An alternative approach}

The experience with the S-scale concerning warnings of elevated levels of radiation at aviation altitudes has led over the last decade to a demand for more specific information due to the unacceptably high number of false alarms generated. This aspect was addressed during an aviation workshop which was held in conjunction with NOAA's Space Weather Workshop in 2012 and the introduction of a new H ("high-energy") scale for radiation, based on readings of the proton flux above $100 \mathrm{MeV}$, was discussed (Meehan \& Kunches 2012). Although the introduction of an increased energy threshold would be in principle a step in the right direction, it should be noted in this context that, even above $100 \mathrm{MeV}$, most of the impinging solar protons and the secondary particles they create do not contribute to the radiation field at aviation altitudes.
Table 1. Space Weather D-index with corresponding solar dose rate contributions $\dot{E}_{\text {sol }}$ given by equation (1).

\begin{tabular}{lc}
\hline \hline Index D & Dose rate interval $[\mu \mathrm{Sv} / \mathrm{h}]$ \\
\hline D0 & $\dot{E}_{\text {sol }}<5$ \\
D1 & $5 \leq \dot{E}_{\text {sol }}<10$ \\
D2 & $10 \leq \dot{E}_{\text {sol }}<20$ \\
D3 & $20 \leq \dot{E}_{\text {sol }}<40$ \\
D4 & $40 \leq \dot{E}_{\text {sol }}<80$ \\
D5 & $80 \leq \dot{E}_{\text {sol }}<160$ \\
D6 & $160 \leq \dot{E}_{\text {sol }}<320$ \\
D7 & $320 \leq \dot{E}_{\text {sol }}<640$ \\
D8 & $640 \leq \dot{E}_{\text {sol }}<1280$ \\
\hline
\end{tabular}

For this reason, we propose the introduction of a new Space Weather index D for warnings of elevated radiation levels. This index is based on dose rates produced by solar protons at aviation altitudes during solar radiation storms and constitutes a relevant parameter for the assessment of their contribution to the corresponding radiation exposure. If the proposed index can be assessed in near real-time it will gain wider acceptance for operational purposes among responsible airline managers and radiation protection officers than an index based on the integral flux of solar protons outside the Earth's atmosphere. In order to cover an ideally wide extent of the solar contribution to the radiation exposure and to be represented by a comparatively small natural number, the Space Weather index D is graduated by a 2-base logarithmic scale of corresponding dose rates and defined as the smallest natural number, including zero, to satisfy the inequality:

$$
\dot{E}_{\text {sol }}<5 \frac{\mu S v}{h} \times 2^{D}
$$

where $\dot{E}_{\text {sol }}$ is the additional contribution by solar protons to the rate of the effective dose, which can be assessed both by measurements of the ambient dose equivalent rate and model calculations (ICRU 2010). The indices from D0 up to D8 and the associated ranges of dose rates are listed in Table 1. This graduation also facilitates the implementation of a mitigation scheme for a proportionate response to the increased radiation exposure during a strong solar radiation storm.

An important feature of the Space Weather index D is its utilization for local warnings, since it is based on the dose rate at a particular point in the Earth's atmosphere, i.e. the D-index is dependent on geographic position and flight altitude. This permits 

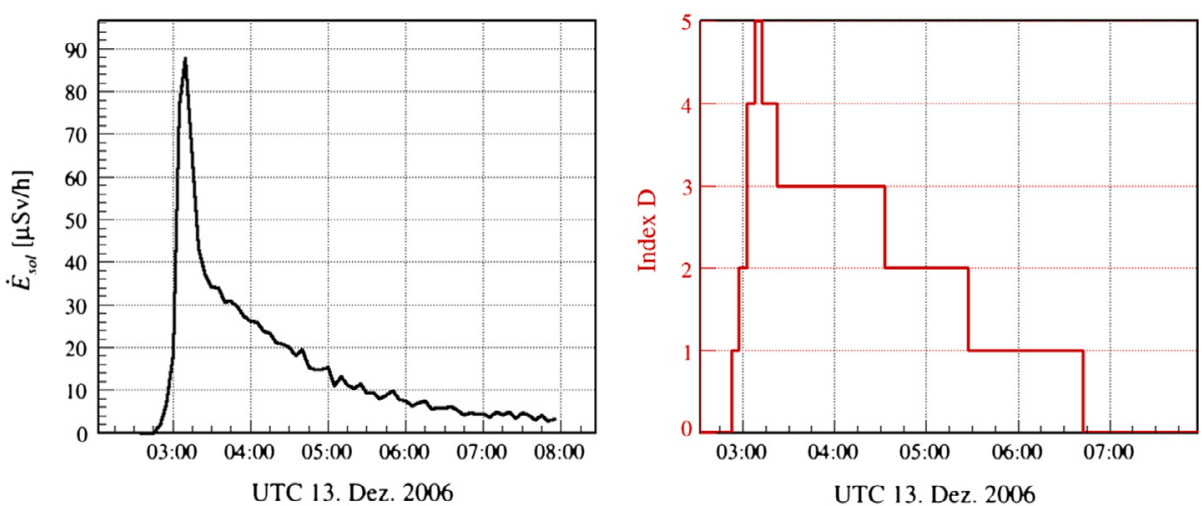

Fig. 3. Temporal profile of the dose rate caused by solar protons $\dot{E}_{\mathrm{sol}}$ at $70 \mathrm{~N}, 50 \mathrm{E}$ at an altitude of $41,000 \mathrm{ft}$. (FL410) assessed with the PANDOCA model during GLE70 on 13th December 2006 (left) and the corresponding D-index (right).
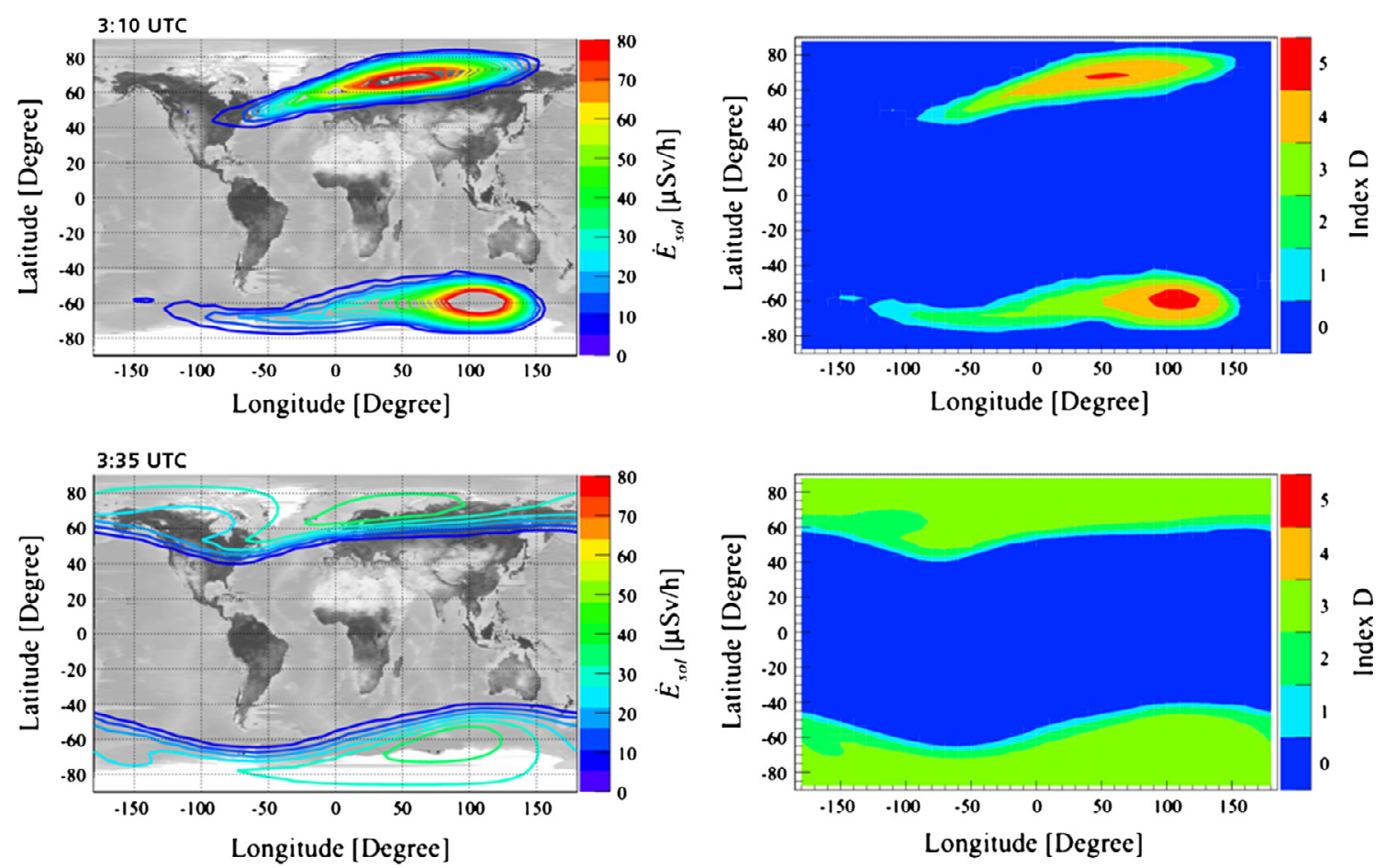

Fig. 4. World map showing areas of increased dose rates due to solar protons represented by contour lines (top left) and the areas of the corresponding D-indices (top right) at maximum exposure which was reached at 03:10 UTC, about 15 min after the onset of the event. The initial hot spots had leveled out according to the geomagnetic latitude (bottom) at 03:35 UTC, about 40 min after the onset of the event, and the derived global $\mathrm{D}_{\mathrm{G}}$-index declined from $\mathrm{D} 5$ to $\mathrm{D} 3$.

a more specific assessment of the effect of a solar radiation storm on particular geographic regions. In order to describe the radiation field in a particular area using a single, generalised local $\mathrm{D}_{\mathrm{L}}$ index, the dose rate is assessed for the upper airspace of civil aviation, i.e. at $41,000 \mathrm{ft}$. (FL410, $12.5 \mathrm{~km}$ ), as a worst case scenario. In addition, a global $\mathrm{D}_{\mathrm{G}}$ index can be derived from the local indices and defined by the maximum local $\mathrm{D}_{\mathrm{L}}$ index anywhere in the world. Thus, a global warning indicating that there is at least one affected area can be issued. A further advantage of the dose rate based Space Weather D-scale is the possibility to assess the radiation exposure for affected flights individually by their routes and flight altitudes. Consequently, an individual $\mathrm{D}_{\mathrm{F}}$-index for the maximum exposure during a particular flight can be derived for making a comparison with the corresponding local and global D-indices.

The threshold of the first stage of the Space Weather index $\mathrm{D}$ is an increase in dose rate by solar protons of $5 \mu \mathrm{Sv} / \mathrm{h}$ which corresponds to about $50 \%$ of the dose rate due to the galactic component in the upper airspace in the polar regions during solar minimum (Hubiak 2008; Meier et al. 2009). A temporary increase below this threshold is of the same order of magnitude as the natural variation of this galactic contribution to the radiation field. Furthermore, the first values on the D-scale have an easily understandable and communicable meaning: D1 indicates a temporary additional solar contribution which is of the order of the exposure to the omnipresent galactic component of cosmic radiation. An index of D2 corresponds to an increase in radiation of about twice the galactic component and D3 represents the dose rate threshold for the radiation alert used by the FAA's Solar Radiation Alert System. In this context it should be noted that such an alert is not triggered until this or a higher level is reached for each of three consecutive 5-min periods (Copeland et al. 2009).

The application of the Space Weather index D can be demonstrated for instance with GLE70 which took place on 
13th December 2006. The corresponding calculations were performed with the PANDOCA model (Matthiä et al. 2009, 2014). The temporal dose profile of the solar contribution to the radiation field $\dot{E}_{\text {sol }}$ and the global index $\mathrm{D}_{\mathrm{G}}$ derived at the geographical position of maximum exposure at a flight level of $41,000 \mathrm{ft}$. (FL410, $12.5 \mathrm{~km}$ ) are shown in Figure 3 with a time resolution of $5 \mathrm{~min}$. After a rapid onset of the increase in dose rate, a maximum was reached at around 3:10 UTC with a corresponding D5-alert which fell off to a D3-alert within some $15 \mathrm{~min}$ and thereafter fell below the lowest warning threshold to D0 about $3.5 \mathrm{~h}$ later. The anisotropic character of the event during the initial phase at 3:10 UTC is represented in Figure 4. While there were spots of increased dose rates with corresponding local indices up to D5 over Russia, Northern Europe and Antarctica, North America was hardly affected at that time. Within the following half hour these spots leveled out according to their geomagnetic latitudes and the derived global $\mathrm{D}_{\mathrm{G}}$-index declined from D5 to D3.

\section{Conclusion}

The dose rate based Space Weather index D for the assessment of the radiation field at aviation altitudes and the issuing of corresponding alerts provides an essential improvement above the current situation characterized by the application of the NOAA S-scale, which was actually developed for other purposes and does not account for the appropriate proton energy range due to atmospheric shielding. Therefore many false alarms were previously issued in the context of an action level based on an S3-alert, e.g. false positive alarms on 24th January 2012 and 7th March 2012 (no GLE) and a false negative alarm during GLE70 when only an S2-alert was issued (Matthiä et al. 2009).

The dose rate based Space Weather Index D can be determined using measurements or model calculations. Several models which permit a near real-time assessment are already available or under development, e.g. PANDOCA (Matthiä et al. 2014), Solar Radiation Alert System (SRA; Copeland et al. 2009) and NAIRAS (Mertens et al. 2013).

The FAA issues a radiation alert with their SRA on the condition that a threshold of $20 \mu \mathrm{Sv} / \mathrm{h}$ at any altitude between $30,000 \mathrm{ft} .(9.1 \mathrm{~km})$ and $70,000 \mathrm{ft} .(21.3 \mathrm{~km})$ for each of three consecutive 5-min periods is exceeded. This would correspond to at least a global D3 warning on the D-scale for $15 \mathrm{~min}$, if the airspace at or below $41,000 \mathrm{ft}$. $(12.5 \mathrm{~km})$ were affected as well. In this context, it is worth mentioning that an SPE which causes an increase in dose rates of $20 \mu \mathrm{Sv} / \mathrm{h}$ or even more at $70,000 \mathrm{ft}$. does not necessarily significantly affect the radiation field at the cruising altitudes of civil aviation, i.e. below FL410 $(41,000 \mathrm{ft}$.), due to the additional atmospheric shielding present, if the energy spectrum of the impinging particles is comparatively soft.

In addition to issuing an alert if a previously set threshold is exceeded, the index $\mathrm{D}$ is configured to give information on the range of the corresponding dose rates, i.e. providing an upper limit.

Recommendations for action during a strong solar particle event can be based on the Space Weather index D in combination with the time spent or likely to be spent in the corresponding radiation field, for instance $1 \mathrm{~h}$ spent in a field with a D3 index would bring about approximately the same radiation dose as $2 \mathrm{~h}$ spent in a field with a D2 index. Irrespective of threshold effects, the reduction of one stage on the D-scale corresponds to reducing the dose rate due to solar protons by about a factor of two, which can be easily communicated to airlines and to the public. A further advantage is the improved visibility of the effects of mitigation measures, such as rerouting and lowering flight altitudes, provided by the corresponding reduction in the $\mathrm{D}$-index for individual flights.

\section{References}

Beck, P., M. Latocha, S. Rollet, and G. Stehno, TEPC reference measurements at aircraft altitudes during a solar storm, Advances in Space Research, 36, 1627-1633, 2005.

Copeland, K., H.H. Sauer, and W. Friedberg, Solar Radiation Alert System, DOT/FAA/AM-05/14, Office of Aerospace Medicine, Washington, DC, 2005.

Copeland, K., H.H. Sauer, and W. Friedberg, Solar Radiation Alert System (revised 30 May 2008), DOT/FAA/AM-09/6, Office of Aerospace Medicine, Washington, DC, 2009.

Getley, I.L., Observation of solar particle event on board a commercial flight from Los Angeles to New York on 29 October 2003, Space Weather, 2, S05002, DOI: 10.1029/2003SW000058, 2004.

Hubiak, M., Experimentelle Bestimmung von Dosisraten auf Reiseflughöhen im solaren Minimum, Diploma-Thesis, Fachhochschule Münster University of Applied Sciences, Germany, 2008.

ICRU, Reference data for the validation of doses from cosmicradiation exposure of aircraft crew, ICRU Report No. 84, J. ICRU, 10 (2), 2010.

Lieber, R., Solar storm rekindles concern over whether radiation hurts fliers, The Wall Street Journal, available at http:// online.wsj.com/news/articles/SB106744850896766200 (accessed 14th January 2014), 2003.

Matthiä, D., B. Heber, G. Reitz, L. Sihver, T. Berger, and M. Meier, The ground level event 70 on December 13th, 2006 and related effective doses at aviation altitudes, Radiat. Prot. Dosimetry, 136 (4), 304-310, 2009.

Matthiä, D., M.M. Meier, and G. Reitz, Numerical calculation of the radiation exposure from galactic cosmic rays at aviation altitudes with the PANDOCA core model, Space Weather, 12, 161-171, DOI: 10.1002/2013SW001022, 2014.

Meehan, J., and J. Kunches, Workshop Addresses Aviation Community, Space Weather, 10, S08011DOI: 10.1029/2012SW000828, 2012.

Meier, M.M., M. Hubiak, D. Matthiä, M. Wirtz, and G. Reitz, Dosimetry at aviation altitudes (2006-2008), Radiat. Prot. Dosim., 136 (4), 251-255, 2009.

Mertens, C.J., M.M. Meier, S. Brown, R.B. Norman, and X. Xu, NAIRAS aircraft radiation model development, dose climatology, and initial validation, Space Weather, 11, 603-635, DOI: 10.1002/swe.20100, 2013.

Poppe, B., New scales help public, technicians understand space weather, Eos, Transactions, American Geophysical Union, 81 (29), 322-328, 2000.

Poppe, B., and K. Jorden, Sentinels of the Sun, Johnson Books, Boulder, ISBN 1-55566-379-6, 2006.

US Department of Commerce, Service Assessment, Intense Space Weather Storms October 19-November 07, 2003, 17-18, available at http://www.swpc.noaa.gov/Services/SWstorms_assessment.pdf (accessed 14th January 2014), 2004.

Cite this article as: Meier MM \& Matthiä D: A space weather index for the radiation field at aviation altitudes. J. Space Weather Space Clim., 2014, 4, A13. 